\title{
Heterotopic Ossification in the Gallbladder
}

\author{
Jihyun Ahn · Sunyoung Kim · Kangseung Kim ${ }^{1}$ Seogjoon Kim² \\ Departments of Pathology, ${ }^{1}$ General Surgery, and ${ }^{2}$ Radiology, Dongkang Medical Center, Ulsan, Korea
}

Heterotopic ossification is a common pathologic process in cases of atherosclerotic plaques, traumatic injury, severe burns, some carcinomas, and benign neoplasms. ${ }^{1}$ However, heterotopic ossification is extremely rare in the gallbladder. Less than 10 cases have been reported in the literature to date. ${ }^{2-8}$ While the pathogenesis of heterotopic ossification of the gallbladder is unclear, it may clinically lead to the misinterpretation of cholelithiasis, etc. Herein, we report a case of heterotopic ossification in the gallbladder epithelium.

\section{CASE REPORT}

A 26-year-old man visited our hospital for abdominal pain of several days duration. Laboratory data showed positivity for hepatitis B surface antigen and hepatitis B e antigen, negativity for hepatitis B surface antibody and hepatitis B e antibody, and increased hepatitis B virus DNA. Alanine transaminase was slightly increased (65 U/L), but aspartate transaminase, bilirubin, alkaline phosphatase, and $\gamma$-glutamyl transpeptidase were within normal range. An ultrasound revealed diffuse wall thickening of the gallbladder with a $1.0 \mathrm{~cm}$-sized gallstone and chronic liver disease (Fig. 1). Computed tomography demonstrated a collapsed gallbladder with mild wall thickening. The patient underwent laparoscopic cholecystectomy. During the operation, a $1.0 \mathrm{~cm}$-sized, black stone was noted in the gallbladder. On gross examination, the gallbladder measured $6.7 \times 3.3 \times 2.0 \mathrm{~cm}$ in size. The mucosal surface was greenish, velvety with focal whitish streaks at the body, about $0.6 \times 0.6 \mathrm{~cm}$ in dimension; in

\section{Corresponding Author}

Jihyun Ahn, MD

Department of Pathology, Dongkang Medical Center, 239 Taehwa-ro, Jung-gu, Ulsan 44455, Korea

Tel: +82-52-241-1375, Fax: +82-52-241-1366, E-mail: jvcjh@hanmail.net

Received: January 28, 2016 Revised: March 4, 2016

Accepted: March 10, 2016 addition, the wall was thickened, measuring up to $1.0 \mathrm{~cm}$. Microscopically, the gallbladder wall was thickened with Rokitansky-Aschoff sinuses and infiltrated by polymorphous inflammatory cells. The mucosa was slightly atrophic with focal mature lamellar bone. Osteoblastic rimming or bone marrow components were absent (Fig. 2).

\section{DISCUSSION}

Heterotopic ossification (bone metaplasia) in the gastrointestinal tract is rare. ${ }^{9}$ Most reported cases were associated with benign and malignant epithelial neoplasms. In the gallbladder, stromal osseous metaplasia has been described in metastatic adenocarcinoma. ${ }^{10}$ However, bone metaplasia in benign gallbladder is extremely rare. In 1957, Indyk and Shipton ${ }^{2}$ described heterotopic bone formation in the gallbladder accompanied by cholelithiasis. Since then, six cases have been reported in the English literature, ${ }^{2-8}$ of which four cases were associated with cholelithiasis and two cases showed spicules of bone in the cholesterol polyps. ${ }^{3,4}$ Rege and Vargas ${ }^{5}$ reported intramuscular fasciitislike proliferation and bony metaplasia in the gallbladder of a 7 -year-old boy with sickle cell disease. They proposed the term cholecystitis ossificans to describe their case; furthermore, they suggested that the mucosal insult by repetition predisposed the gallbladder to dystrophic calcification and/or fasciitis, subsequently creating a favorable microenvironment for bone formation. ${ }^{5}$ This mechanism is further corroborated by reports of heterotopic ossification in other sites of chronic injury, such as atherosclerotic plaques, traumatic injury, and severe burns. However, it is still early to conclude that stones induce heterotopic ossification in the gallbladder.

Three other cases showed heterotopic ossification without accompanied cholelithiasis. Chen ${ }^{6}$ described a case of polypoid intramucosal lesions composed of bone lined by flattened gall- 
bladder mucosa, designated as osteoma of the gallbladder. Yosepovich et al. ${ }^{7}$ and Nelson and $\mathrm{Kahn}^{8}$ also described heterotopic bones occupying gallbladder wall or mucosa, respectively.

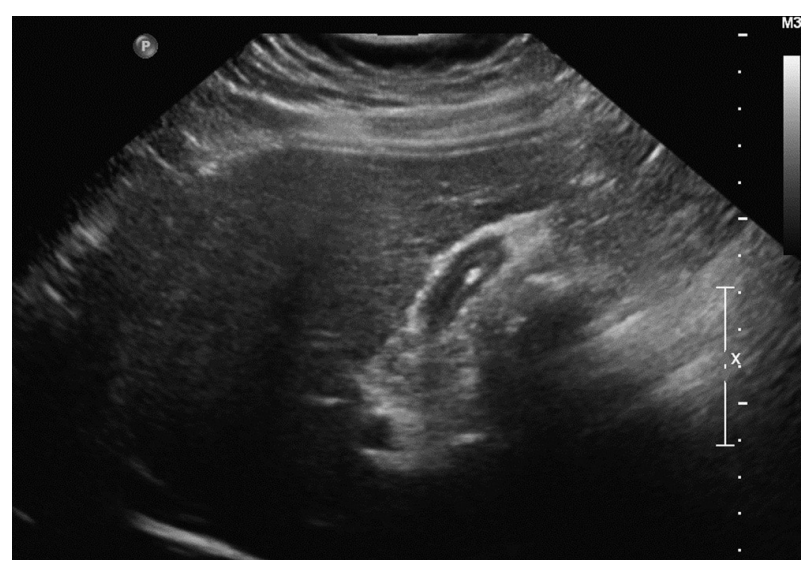

Fig. 1. Radiologic finding. Ultrasound shows diffuse wall thickening of the gallbladder with a $1 \mathrm{~cm}$-sized gallstone.
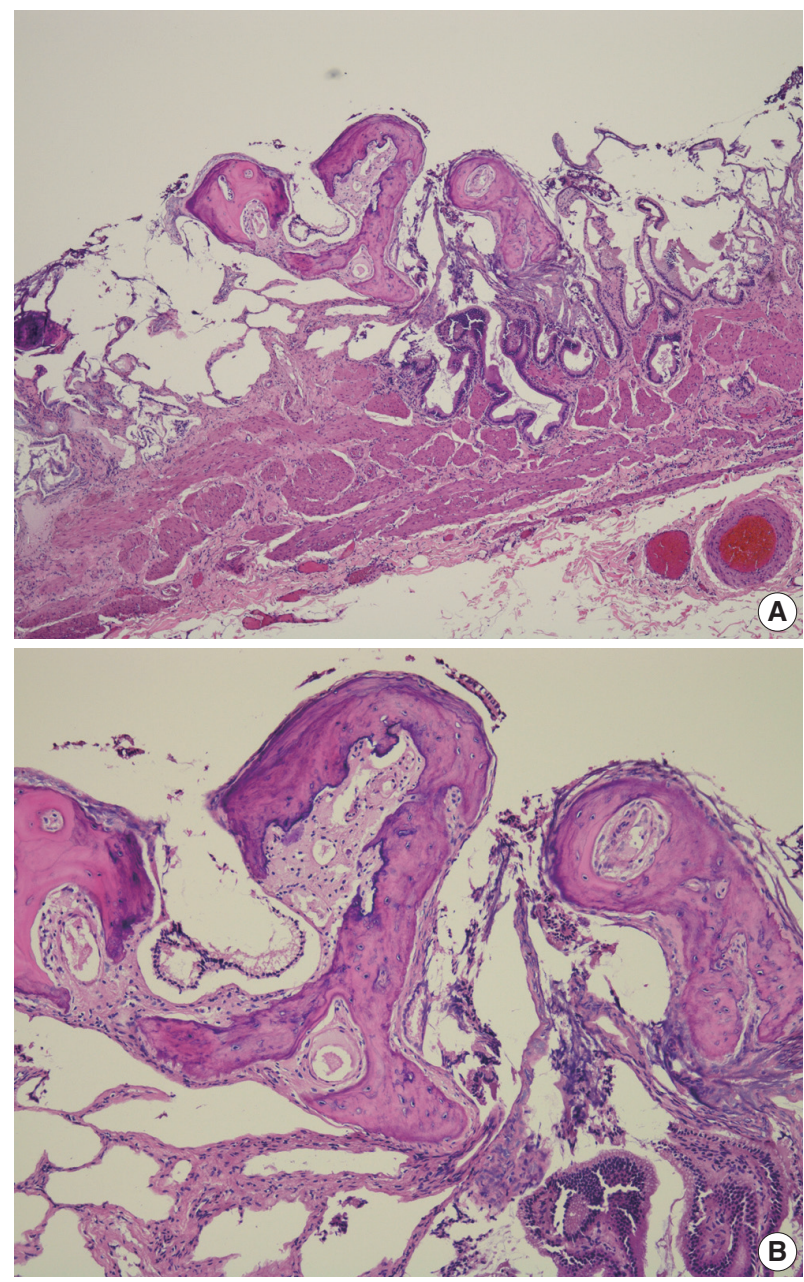

Fig. 2. Pathologic findings. (A, B) Microscopic findings show autolytic mucosa with focal mature bone.
The former was composed of mature lamellar bone with osteoblastic rimming and bone marrow components, while osteoblastic rimming or bone marrow was absent in the latter. ${ }^{7,8}$ Our case was similar to the latter except for the occurrence of the stone. Clinicians should be aware of heterotopic ossification since it can clinically mimic stones and lead to altered treatments and patient outcome.

To our knowledge, this is the first report of heterotopic ossification in the gallbladder in Korean patients. The pathogenesis and clinical significance of heterotopic ossification remain to be elucidated.

\section{Conflicts of Interest}

No potential conflict of interest relevant to this article was reported.

\section{REFERENCES}

1. Ramirez DM, Ramirez MR, Reginato AM, Medici D. Molecular and cellular mechanisms of heterotopic ossification. Histol Histopathol 2014; 29: 1281-5.

2. Indyk JS, Shipton EA. Heterotopic bone formation in the gall-bladder. Med J Aust 1957; 44: 9-11.

3. Ortiz-Hidalgo C, Baquera-Heredia J. Osseous metaplasia in polypoid cholesterosis. Am J Surg Pathol 2000; 24: 895.

4. Lin MY, Shikle JF. To pick a 'bone' with the gallbladder. South Med J 2009; 102: 773-4.

5. Rege TA, Vargas SO. Cholecystitis and cholelithiasis associated with an intramural fasciitis-like proliferation and osseous metaplasia. Pediatr Dev Pathol 2011; 14: 80-3.

6. Chen KT. Osteomas of the gallbladder. Arch Pathol Lab Med 1994; 118: 755-6.

7. Yosepovich A, Nass D, Zagatsky M, Kopolovic J. Chronic cholecystitis with bone metaplasia: a case report. Pathol Res Pract 2002; 198: 765-6.

8. Nelson JJ, Kahn AG. A case of bone metaplasia of the gallbladder epithelium. South Med J 2009; 102: 322-4.

9. Haque S, Eisen RN, West AB. Heterotopic bone formation in the gastrointestinal tract. Arch Pathol Lab Med 1996; 120: 666-70.

10. Cavazza A, De Marco L, Asioli S, Pastore L, Gardini G. Stromal osseous metaplasia in metastatic adenocarcinoma of the gallbladder. Tumori 1999; 85: 133-4. 